REVISIONES

\title{
Deterioro y preservación de maderas mediante el uso de preservadores naturales de potencial interés en Brasil
}

\author{
Deterioration and preservation of wood by using natural preservatives \\ of potential interest in Brazil
}

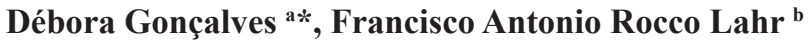 \\ *Autor de correspondencia: ${ }^{a}$ Universidade de São Paulo, Instituto de Física de São Carlos, \\ Av. Trabalhador São-Carlense, 400, 13566-590, São Carlos-SP, Brasil, deborag0405@gmail.com

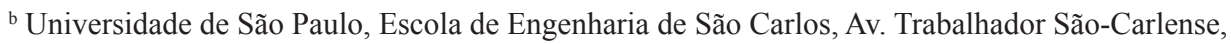 \\ 400, 13566-590, São Carlos-SP, Brasil.
}

\begin{abstract}
SUMMARY
In this review article, the main deterioration processes and uses of natural preservatives in wood are discussed, in particular in Brazil, taking into account its economic relevance in Latin America. It contextualizes the problem of protection and chemical degradation of wood, topics of interest not only in Brazil but also in other countries. In addition, the use of novel products is discussed, such as neem oil, castor oil, pepper extracts, tannins, tall oil, linseed oil, oils extracted from native timbers, chitosan and others. In terms of solutions, more studies are necessary in the field and modification of formulations with fixation agents, biocides, antioxidants, additives and others. In addition, the legal aspects of commercialization for new formulations in countries rich in natural resources, though underdeveloped, must be taken into account.
\end{abstract}

Key words: natural preservatives, natural extracts, tannins, wood deterioration.

\section{RESUMEN}

En este artículo de revisión se discuten los principales procesos de deterioro y del uso de preservadores naturales de la madera, en particular en Brasil, teniendo en cuenta su relevancia en Latinoamérica. Pretende también ser una contribución a los trabajos que tratan de los avances y del desarrollo de nuevas composiciones para preservar maderas. Se contextualiza la problemática de la protección y degradación química de la madera, temas de interés en la actualidad, tanto en Brasil como en otros países. Además, se discute el uso de nuevos productos, tales como aceites de neem y de ricino, extractos de pimientos, taninos, tall oil, aceite vegetal de linaza, aceites esenciales extraídos de maderas nativas y quitosano, y sobre la problemática del bajo uso comercial de productos naturales. En términos de soluciones, hay la necesidad de más estudios de campo y de modificar las formulaciones con agentes de fijación, biocidas, antioxidantes, y aditivos. También hay que tener en cuenta los aspectos legales de la comercialización de nuevas formulaciones en países ricos en recursos naturales pero subdesarrollados.

Palabras clave: preservadores naturales, extractos y aceites naturales, taninos, deterioro de maderas.

\section{INTRODUCCIÓN}

La madera es una materia prima renovable de gran importancia económica en distintos sectores de la sociedad, tales como de la construcción civil, fabricación de muebles y tableros de fibras, y la producción de papel y de la pulpa de celulosa. Sin embargo, existe una creciente preocupación por al menos dos factores. El primero es la tala indiscriminada de especies nativas, particularmente en Brasil, donde existen grandes extensiones de tierras deforestadas y de pérdida de bosques nativos, a pesar del aumento de áreas de plantío (Vidal et al. 2015). Además, las áreas de conserva- ción en Brasil son creadas bajo presiones externas, o sea, sin existir gran preocupación ambiental. El segundo hace referencia a la preservación de maderas, pues los impactos ambientales negativos son producidos por el uso de preservadores tóxicos y por la ubicación, y consecuente eliminación, de maderas tratadas y de residuos generados de las mismas.

El amplio uso de la madera en los sectores de la construcción civil y de muebles ocurrió gracias al avance de dos principales procesos contra el deterioro: el tratamiento térmico y la impregnación con preservadores químicos. La pirólisis mantiene la estabilidad dimensional y mejora la durabilidad de la madera expuesta a los agentes externos y 
a la humedad. Además, el tratamiento a temperaturas bajas (menor de $200^{\circ} \mathrm{C}$ ) puede evitar el deterioro irreversible de la madera (Oliveira et al. 2010). El deterioro térmico de la madera comienza alrededor de $180^{\circ} \mathrm{C}$ con la degradación de las hemicelulosas y la modificación química de la estructura de la lignina (Oliveira et al. 2010). Estos procesos están asociados a cambios de coloración y dimensionales de la madera, ya que ocurre su descomposición química y la aparición de fendas de secado o grietas.

En temperaturas aún mayores, arriba de $\operatorname{los} 325^{\circ} \mathrm{C}$, ocurren cambios significativos en las partes internas de la madera, favoreciendo la volatilización de los extractos y la destrucción, total o parcial, de los componentes individuales (hemicelulosa y lignina) (Kamdem et al. 2002). El preservador químico de la madera, en cambio, actúa contra la acción de los agentes físicos, químicos y biológicos, y favorece el aumento de la resistencia y la durabilidad; además, puede estar vinculado a la reducción de costos mediante el uso más eficiente de los recursos forestales (Kamdem et al. 2002).

\section{DETERIORO DE LA MADERA}

La degradación química de la madera se produce no solo como resultado de la variación de la temperatura, sino también a partir del ataque de hongos y bacterias. Estos diferentes agentes pueden cambiar la composición química, la resistencia mecánica, el color, la permeabilidad y la densidad de la madera en condiciones favorables de humedad y temperatura, en presencia o no de oxígeno (Goodell et al. 2003). Los hongos son responsables por grandes pérdidas de los productos maderables, ya que pueden descomponer la materia orgánica, y metabolizar a los compuestos orgánicos complejos en unidades más pequeñas y digeribles.

Los daños causados por hongos se manifiestan bajo distintas formas de pudrición (Mirski et al. 2014): blan$\mathrm{ca}$, que destruye los pigmentos de la madera y provoca la aparición de fibras sueltas, en el caso de una colonización temprana; marrón, que causa el continuo deterioro de la celulosa y de la hemicelulosa, y conserva casi intacta la lignina; y blanda o suave, que se caracteriza por una baja penetración superficial. El último tipo de colonización convierte la madera, cuando está húmeda, en una masa amorfa y ablandada y que, por lo tanto, se puede llamar pudrición blanda (Mirski et al. 2014).

Los insectos representan otro tipo de organismo xilófago dañino, en gran medida por la severidad del ataque de las termitas en maderas tropicales. Las termitas son insectos sociales, miembros del orden Blattodea, sub orden Isoptera, que se clasifican en cuatro grandes grupos, según sus fuentes de alimentación y hábitos de nidificación: de las maderas húmedas o secas, y subterráneas o arbóreas (Wako 2015). Se conocen más de 2.600 especies de termitas alrededor del mundo, que están en edificios, casas, infraestructuras, bosques y plantaciones forestales (Wako 2015). Las termitas se alimentan de la celulosa de las maderas; pero, como la ce- lulosa es de difícil digestión, su descomposición en azúcar y carbohidratos se debe a los microorganismos presentes en el aparato digestivo de las mismas (Wako 2015).

Cuando la durabilidad natural de la madera es baja frente al ataque de los agentes de deterioro se suelen emplear productos biocidas. Además, existen opciones importantes para disminuir, o impedir, su deterioro, tales como: el uso de especies con alta resistencia natural, la incorporación de productos químicos de superficie, y la modificación química permanente de su estructura mediante el tratamiento térmico. Entre las opciones mencionadas, la fijación del preservador es una alternativa conocida y viable dentro del campo de tecnologías en maderas.

\section{IMPREGNACIÓN DE LA MADERA CON PRESERVADORES SINTÉTICOS}

La impregnación de la madera es un proceso de fijación de productos químicos hasta alcanzar cierta penetración para el interior de la madera, y que puede variar en decenas de milímetros. Este tipo de metodología tiene la finalidad de convertir la madera en un material tóxico y retardar o prevenir el ataque de agentes xilófagos. Los preservadores deben reunir una serie de requisitos: alta penetrabilidad, alta resistencia a los procesos de lixiviación, baja volatilidad, baja o ninguna toxicidad en seres humanos y animales, y relativamente bajo costo.

La fijación química es una de las metodologías más empleadas de preservación de maderas de bosques plantados y se basa en la actuación de los componentes tóxicos presentes en los preservadores contra el deterioro. Los preservadores de maderas más conocidos son solubles en agua y contienen óxidos o sales de cromo, cobre, zinc, boro y arsénico, además de otros componentes (Vidal et al. 2015). Algunos de ellos, tal como el arseniato de cobre cromatado (CCA), son tóxicos por la presencia de metales pesados (cromo y cobre) y arsénico (Hasan et al. 2010). Generalmente, la madera tratada con preservadores sintéticos comerciales libera una cierta cantidad de componentes tóxicos por lixiviación, y que son responsables por la contaminación de suelos y de aguas subterráneas.

El reemplazo gradual de preservadores sintéticos por otros más naturales es una tendencia reciente, ya que implica mejor eficiencia de protección y baja o ninguna emisión de compuestos tóxicos. En este sentido, el interés en preservadores naturales sigue creciendo en países latinoamericanos, ya que muchas de las plantas son reconocidas por sus propiedades pesticidas, insecticidas o fungicidas (Singh y Singh 2012).

\section{EJEMPLOS DE PRESERVADORES COMERCIALES DE MADERAS}

Los preservadores de maderas más conocidos son clasificados según las categorías de origen: oleosos (como creosota), oleosolubles (solubles en solventes oleosos) e 
hidrosolubles (Lepage et al. 2017). Los últimos presentan denominaciones genéricas y son a base de óxidos, ácidos y sales inorgánicos, así como el sulfato de cobre, dicromato de potasio o sodio, ácido crómico y ácido arsénico. La denominación ACA incluye los productos a base de cromo y de arsénico en solución amoniacal; CCA, a base de cobre, cromo y arsénico; y CCB, a base de cobre, cromo y boro. Uno de los procedimientos industriales más utilizados en Brasil es el método Bethell/Burnett, que se basa en la impregnación de maderas con preservadores en autoclave y a presión (Vidal et al. 2015).

Según la normativa brasileña sobre la durabilidad de las maderas en estructuras, NBR 7190, Anexo D, Associação Brasileira de Normas Técnicas - ABNT (1997), los preservadores de acción prolongada son usados en la mayoría de los procesos de impregnación de maderas del mundo. Mientras tanto, algunos preservadores, antes empleados en más amplia escala, tales como el lindano y el pentaclorofenol, están prohibidos en diferentes países como consecuencia de los riesgos de toxicidad, NBR 7190, Anexo D (ABNT 1997). Esta normativa fue revisada y los preservadores fueron clasificados en grupos de riesgo: CCA, CCB, creosota, tribromofenol, boratos, quinolinolato de cobre y carbendazim (Vidal et al. 2015).

En Brasil, hay una gran demanda por los óxidos de CCA tipo $\mathrm{C}$, que garantizan la impregnación de la madera bajo presión. Recientemente, una lista de 41 productos con registro fue actualizada por el Instituto Brasileño del Medio Ambiente y de los Recursos Naturales Renovables, IBAMA (2018). Estos productos comerciales contienen diferentes componentes activos, en su mayoría a base de piretroides, óxidos y sales metálicos (cuadro 1).

La United States Environmental Protection Agency (EPA) restringió el uso de CCA en viviendas, espacios libres para niños, y decks en madera como una medida preventiva (EPA 2003). Otros países, tales como Japón, Indonesia, Suecia, Alemania y Australia, impusieron restricciones parciales o totales al CCA (Vidal et al. 2015), lo que se debe a los problemas producidos por la exposición al cobre y arsénico y por la disposición final de las maderas tratadas con CCA.
Mientras surgió una segunda generación de preservadores, el CCA aún se mantiene como uno de los preservadores químicos más empleados en Brasil, y prácticamente sin restricciones (Vidal et al. 2015). Otros preservadores basados en la cipermetrina y tebuconazol/cobre, de acuerdo con el cuadro 1, son poco empleados en Brasil, teniendo en cuenta las cuestiones de costos sumadas a un perfil más conservador de mercado. La cipermetrina hace parte del grupo de los piretroides y actúa como biocida en la impregnación de la madera. La clasificación toxicológica de la cipermetrina es clase II (muy tóxico, rojo) y ha sido legalizada en Brasil para el uso en postes, columnas, pilares y vigas (ANVISA 2018). O sea, aunque vinculados a problemas de toxicidad, algunos productos químicos libres de metal han sido empleados en Brasil para proteger la madera, como es el caso de las emulsiones basadas en biocidas piretroides sintéticos.

Más recientemente, nanopartículas de plata, boro, cobre, zinc, óxido o borato de zinc, dióxido de titanio y otros han surgido como una apuesta a la nueva generación de preservadores sintéticos de la madera (Borges et al. 2018). En este caso, estos nanomateriales presentaron la capacidad de modificar y proteger la madera, generalmente encapsulados en polímeros (Papadopoulos y Taghiyari 2019). Sin embargo, se reconoce la necesidad de más estudios sobre como los nanomateriales pueden reemplazar a los productos más tóxicos de manera segura y eficiente. Además, es conveniente que sean establecidas las directrices sobre seguridad en el momento de la aplicación de los nanomateriales en la madera. No obstante, los resultados positivos, el consumo de preservadores de maderas en muchos países aún se mantiene respaldado por un perfil más conservador, ya que cambios lentos se han notado si se trata del uso de productos distintos de los usuales.

\section{ALTERNATIVAS PARA LA PRESERVACIÓN DE LA MADERA EN BRASIL}

Las maderas impregnadas por productos químicos pueden liberar ciertas cantidades de componentes tóxicos por lixiviación, así una alternativa es el uso de productos

Cuadro 1. Componentes activos de productos comerciales registrados en Brasil.

Active constituents of commercial products registered in Brazil.

$$
\text { Componentes activos }
$$

Acción

Carbendazim, 8-quinolato de cobre, 2,4,6-tribromofenol (TBP), tanino (Jimo Ecomofo), 3-iodo-2-propinil Fungicida butil carbamato (IPBC)

Creosota, cipermetrina, 2,4,6-tribromofenol, deltametrina, IPBC, CCA, CCB, propiconazol, tebuconazol Insecticida y fungicida y cobre (Tanalith E), boro y fluoreto (Polesaver rods/bandages).

Ciflutrina (Preventol HS)

Insecticida y termiticida

Cipermetrina (Osmose CP), deltametrina (Penetrol Cupim)

Insecticida

Fuente: IBAMA (2018). 
naturales, abundantes, de baja toxicidad y alta fijación. Las iniciativas que buscan el desarrollo de los productos naturales y sostenibles para la protección de maderas han crecido en varios países, incluyendo los aceites esenciales mezclados, los extractos de plantas (canela y linaza) y frutos cítricos, las ceras y las resinas de la corteza de diferentes especies arbóreas (Singh y Singh 2012). Algunos de los productos naturales más utilizados en Brasil en los últimos años serán presentados en esta revisión.

Aceites de neem y de ricino. Los aceites de neem (Azadirachta indica A. Juss.) y de ricino (Ricinus communis L.) son especialmente estudiados en Brasil. El aceite de neem debe su actividad insecticida a la presencia de un ingrediente activo principal, un limonoide, azadirachtina, que induce la mortalidad entre los insectos (Viana et al. 2006). La literatura señala que el aceite de neem actúa de manera efectiva en la madera de ceiba (Ceiba pentandra (L.) Gaertn.), especie típica de florestas tropicales abiertas, cuya madera es muy atacada por hongos e insectos (Paes et al. 2012). Las mejores concentraciones de aceite de neem usadas en pino (Pinus radiata D.Don) y ceiba se mostraron elevadas (50\%), lo que suele explicar que una protección más durable y efectiva contra las termitas y los hongos no debe ocurrir en concentraciones más bajas (Machado et al. 2013). Además de haber la necesidad del uso de más altas concentraciones de aceite de neem, las maderas tratadas con este aceite, y en contacto con el suelo, sufrieron deterioro con el tiempo; por eso, la propuesta de optimización ha sido el uso del aceite de neem junto a co-biocidas reforzantes (Machado et al. 2013). A pesar del interés en el aceite de neem como agente insecticida, biopesticida y también por propiedades terapéuticas (anticáncer y microbicida) (Fernandes et al. 2019), pocos estudios recientes se dedican a estudiar más detalladamente su perfil como preservador de maderas.

El aceite de ricino ha sido añadido al aceite de neem como un tensoactivo en la búsqueda de mejores propiedades anti-hongos y anti-bacterias (Machado et al. 2006), y también como un adhesivo en tableros de fibras de densidad media (MDF) y preparados con residuos de Pinus spp. tratados con CCB (Bertolini et al. 2013). El aceite de ricino contiene cerca de $90 \%$ de ácido ricinoleico, un ácido graso insaturado soluble en etanol a bajas temperaturas, viscoso y con propiedades de interés para la producción de biodiesel en Brasil. Se ha verificado también que el aceite de ricino asegura una mejora en la resistencia de Ceiba contra las termitas subterráneas (Nasutitermes corniger Motschulsky) por medio de un menor porcentaje de pérdida de masa (Paes et al. 2011, 2012). Sin embargo, la protección de la madera envejecida por medio de la volatilización del aceite de ricino no se mostró tan duradera (Paes et al. 2011). Estudios más recientes han destacado el uso de las resinas de poliuretano obtenidas a partir del aceite de ricino en revestimientos de MDF, que pasan a retener menos agua y a presentar más baja capacidad de hinchamiento, tanto en espesura cuanto en volumen (Calegari et al. 2017).

Taninos. Los taninos son extractos vegetales presentes en las hojas, frutos, semillas y corteza de una gran variedad de especies arbóreas (Shirmohammadl et al. 2018). Además, son asignados de acuerdo a dos grupos principales, los hidrolizables y los condensados, y se presentan como compuestos polifenólicos de valores de masa molar relativamente altos (Nascimento et al. 2013). Sus principales aplicaciones son en el proceso de curtido de piel, en la industria farmacéutica, en el tratamiento de aguas y en la formulación de adhesivos (Calegari et al. 2014). Los taninos condensados son conocidos por sus propiedades antihongos y actúan como preservadores atóxicos y con una gran capacidad de formar complejos con iones metálicos (Calegari et al. 2014). Nuevas formulaciones han sido evaluadas teniendo en cuenta la propiedad quelante del tanino con el boro y el cobre, por ejemplo, que son componentes tóxicos para las lignocelulosas de la madera (Calegari et al. 2014). Mientras tanto, una cuestión importante es que el uso de taninos no es recomendable en ambientes externos, una vez que son solubles en agua; en este caso, los mismos pueden ser modificados con otros compuestos químicos para disminuir la lixiviación (Calegari et al. 2014).

Trabajos recientes señalan el papel de los taninos condensados de árboles brasileños contra las termitas y los hongos (Calegari et al. 2014, Silveira et al. 2017). Estos fueron obtenidos a partir de las especies jurema-preta ( $\mathrm{Mi}$ mosa tenuiflora (Willd.) Poir.) (Calegari et al. 2014) y acacia-negra (Acacia mearnsii De Wild.) (Silveira et al. 2017) y se mostraron más efectivos cuando mezclados al ácido bórico a $2 \%$ (Calegari et al. 2014). El uso de taninos $5 \%$ y $10 \%$ llevó a resultados similares a los obtenidos con el CCB frente al ataque de Pycnoporus sanguineus (L.) Murrill en la especie Acacia mearnsii (Silveira et al. 2017).

Los extractos obtenidos del leño y la corteza de especies de la floresta Amazonia brasileña también presentaron propiedades repelentes a las termitas del género Nasutitermes Dudley (Barbosa et al. 2007). Entretanto, el desempeño de los taninos en áreas externas fue considerado peor que los obtenidos con los preservadores comerciales, pero suelen ser utilizados en áreas internas y más secas, y externas protegidas (Tomak y Gonultas 2018). Otra cuestión relevante es la ventaja del uso de taninos comerciales, tales como aquellos empleados en la industria del curtimiento de pieles, sobre todo porque están bien caracterizados y presentan pureza conocida en su composición (Calegari et al. 2014).

Extractos de pimientos y de partes de árboles. El género Capsicum incluye una gran variedad de pimientos picantes, cuyos principales atributos sensoriales son color, textura y picor. Los compuestos responsables del picor de los pimientos son los capsaicinoides y diferentes compuestos han sido identificados en este grupo; sin embargo, el con- 
tenido de la capsaicina es la responsable por el picor de los pimientos (Ziglio et al. 2018). Resultados obtenidos a escala de laboratorio indicaron que la oleorresina de un pimiento picante (Capsicum frutescens Mill, "Bhut jolokia") se mostró más efectiva en la protección de Pinus sp. y Hymenaea sp. contra al ataque del hongo de pudrición blanda, Paecilomyces variotii Bainier (Ziglio et al. 2018). No obstante, son necesarios más estudios en lo que concierne a los efectos de la impregnación de la madera con extractos de pimientos, y las relaciones biodegradacióntiempo y costo-beneficio.

Los extractos obtenidos de partes y de residuos de árboles son otras alternativas contra el ataque de los hongos y las termitas en las maderas. Los extractos de la madera teca (Tectona grandis L.f.) han sido empleados para proteger superficies de teca (albura) y Pinus sp. contra el ataque de hongos de pudrición blanca y marrón mediante ensayos de deterioro acelerado (Brocco et al. 2017). También se han realizado estudios acerca del deterioro acelerado de teca impregnada con extractos de teca contra el ataque de las termitas, permitiendo una mejoría en el color de la madera (Brocco et al. 2020). La fijación de los extractos de teca promovió un aumento en la resistencia de las maderas de teca a hongos de pudrición blanca o marrón en valores de pérdidas de masa. En este caso, las maderas fueron clasificadas como resistentes y altamente resistentes al ataque de hongos (Brocco et al. 2017). Mientras tanto, a pesar de los resultados positivos, poco se conoce acerca de la ecotoxicidad y de la viabilidad técnica y económica de los preservadores de maderas a base de los extractivos de la madera teca (Brocco et al. 2017). Hace algunas décadas el cultivo de la teca se ha desarrollado en Brasil de tal manera que esta especie se ha adaptado bien a las condiciones climáticas tropicales hasta alcanzar baja edad de rotación de plantío (Florez et al. 2014). Así, los extractos de teca apuntan como una alternativa de interés para el uso como preservadores de maderas.

Aceites esenciales extraídos de maderas nativas. Los aceites esenciales extraídos de maderas nativas del Cerrado brasileño (Lippia origanoides Kunth y Lippia lacunosa Mart. et Schauer) y del clavo de olor (Syzygium aromaticum (L.) Merr. et L.M.Perry) fueron empleados en diferentes concentraciones $(12,5 \%, 25 \%, 50 \%$ y $100 \%)$ contra hongos Gloeophyllum trabeum (Pers.) Murrill y Trametes versicolor (L.:Fr.) Quél. (Medeiros et al. 2016). $\mathrm{El}$ aceite esencial de L. origanoides se mostró más efectivo contra el ataque del hongo en todas las concentraciones estudiadas por la presencia de altos contenidos de timol.

La acción contra los hongos fue relacionada a la presencia de compuestos fenólicos (eugenol - clavo, y timol) en los aceites esenciales, que afectan sus interacciones con la superficie de la madera por medio de diferencias de polaridad (Cheng et al. 2008). Una vez que timol presenta una toxicidad relativamente baja para los seres humanos y la naturaleza, y se produce a $2,92 \%$ de rendimiento de extracción - considerado alto, el aceite de L. origanoides apunta como un preservador antifúngico con potencial de aplicación en la madera (Medeiros et al. 2016).

Trabajos más recientes tratan del empleo de aceites de especies forestales comúnmente encontradas en Brasil - andiroba (Carapa guianensis Aubl.), copaiba (Copaifera langsdorffii L.) y jatrofa (Jatropha curcas (L.) Britton et Millsp.) (Sousa et al. 2018, Freitas et al. 2019) contra el ataque de las termitas de la madera seca (Cryptotermes brevis Walker). Los aceites estudiados han sido enriquecidos con $\mathrm{I}_{2}(1 \%, 3 \%$ y $5 \%)$ y fueron aplicados en Pinus elliottii Engelm. La elección del $\mathrm{I}_{2}$ se debe a sus propiedades bactericidas, bacteriostáticas y fungicidas, además de su baja solubilidad en agua (Ihssen et al. 2014), una ventaja para frenar, o retardar, los efectos de lixiviación. Las maderas han sido impregnadas con los preservadores por inmersión a frío logrando una eficiente inhibición de crecimiento del hongo (Sousa et al. 2018). Los aceites de andiroba con $\mathrm{I}_{2}$ al $3 \%$ y $5 \%$ y jatrofa con $\mathrm{I}_{2} 5 \%$ llevaron a la total mortalidad de las termitas (Freitas et al. 2019).

Aceites vegetales - linaza, jojoba y soya. Los aceites vegetales de linaza (Linum usitatissimum L.) y jojoba (Simmondsia chinensis (Link) C. K. Schneid.) han sido empleados en la protección, decoración y la limpieza de maderas. El aceite de linaza es considerado uno de los mejores productos naturales para la impregnación de la madera por cuenta de su poder de secado, lo que proporciona impermeabilidad y protección (Bossardi y Barreiros 2011). Comercializado en Brasil, el aceite de linaza actúa como un componente activo en pintura, barniz y tinte, pero es más efectivo en presencia de aditivos para mejorar el secado, y no en el su estado bruto. Otro aceite, de soya, ha sido relacionado a una disminución de la aparición de grietas en Pinus sp. termotratado, lo que mejoró la estabilidad dimensional (Zablonsky et al. 2017).

Aceite de resina, TOC. El aceite de resina (tall oil crudo, TOC) se ha destacado como materia prima en la industria química y en la producción del biodiesel. El TOC es un líquido viscoso, marrón oscuro y obtenido de la industria de celulosa (proceso Kraft). La presencia de ácidos grasos garantiza al TOC sus propiedades hidrofóbicas y esto favorece su aplicación como preservador de la madera, una clave en la mejora de la resistencia al agua de fibras celulósicas (Hosseinpourpia et al. 2018). En este caso, una vez reducido el tenor de humedad de la madera se debe disminuir la capacidad de avance del desarrollo de los hongos sobre la superfície (Dias y Barreiros 2017a). El aceite eyector (ejector oil, EO) es un subproducto del fraccionamiento del TOC y ha sido empleado como preservador de Pinus elliotti Engelm. y Eucalyptus grandi W.Hill ex Maiden. contra el ataque de hongos de pudrición blanca (Dias y Barreiros 2017b). El EO substituyó el TOC con más eficiencia y se observó una mejor impregnación del EO en medidas de pérdidas de masa de las maderas sometidas a 
pudrición acelerada. No obstante los resultados de interés, hay pocos estudios sobre el papel de los aceites de resina en la impregnación de maderas, aunque apuntan direcciones para la producción de nuevas formulaciones.

Biopolímeros - quitosano. Otros productos alternativos para el uso como preservadores de maderas son los geles a base de quitosano en soluciones de ácido acético y que fueron empleados en cajas de maderas de transporte de alimentos (Pinus sp.) (Sattolo et al. 2010). Los geles de quitosano arrojaron resultados prometedores, aunque preliminares, contra el ataque de hongos en alta humedad relativa (Sattolo et al. 2010). El quitosano puede ser obtenido por medio del tratamiento de la quitina, que está presente en grandes cantidades en crustáceos e insectos, y tiene conocida acción biocida. Brasil es uno de los grandes productores de quitosano de origen animal siendo muy estudiado por su versatilidad de aplicación en diferentes sectores.

\section{PERSPECTIVAS DEL USO DE PRESERVADORES NATURALES EN BRASIL DENTRO DEL ESCENARIO GLOBAL}

Aunque resultados prometedores han sido presentados acerca de los preservadores naturales de la madera, están más restringidos a los laboratorios, o sea, en pequeña escala y lejos de los procesos de producción industrial. La eficiencia del preservador de la madera va a depender esencialmente de su capacidad de impregnación y de fijación, tiempo de retención y lixiviación. Lo que concierne a la madera, depende da la especie, el corte, la edad, las condiciones de desarrollo y su ambiente interno o externo. De esta manera, son necesarios más estudios de campo, abarcando una gama más amplia de variables experimentales para hacer llegar el producto al mercado, por ejemplo: estudiar la posibilidad de modificar las formulaciones con agentes específicos (fijantes, biocidas, antioxidantes y aditivos), usar diferentes métodos de fijación, considerar los problemas de lixiviación y del uso en ambientes exteriores, la relación costo-beneficio y las cuestiones legales de comercialización. No hay un plan de apoyo a las industrias en Brasil, tampoco interés centrado en ampliar las investigaciones en materiales resinosos, como ejemplo. Con respecto al incremento de la elaboración de nuevos productos competitivos en el mercado brasileño, es notorio el bajo número de estudios en campo y que vislumbren cambios y soluciones comerciales. Mientras estos nuevos productos pueden promover el aumento de la resistencia biológica y de la durabilidad de la madera, todavía hay mucho por investigar acerca de la retención, biodegradación (estabilidad), eficiencia, costo, diferencias de formulación y métodos de impregnación (Singh y Singh 2012). Hoy la web cuenta con sitios relevantes para obtener información acerca del desarrollo y lanzamiento de formulaciones, tales como: Agência Nacional de Vigilância Sanitária
(ANVISA), International Research Group on Wood Protection (IRG), United States Environmental Protection Agency (EPA), Instituto Brasileiro do Meio Ambiente e dos Recursos Naturais Renováveis (IBAMA) y Embrapa Florestas (Brasil).

\section{CONCLUSIONES}

El uso de preservadores naturales de la madera apunta como una alternativa para disminuir o eliminar los problemas ambientales y de salud causados por los preservadores sintéticos comerciales. Muchos de los componentes químicos presentes en los preservadores comerciales son tóxicos y pueden generar contaminación ambiental. Las alternativas más promisorias para sustituir los preservadores sintéticos son los extractos vegetales y los aceites esenciales mezclados, que pueden ser encontrados en mayor abundancia en la flora brasileña. La materia prima es abundante en Brasil, que es uno de los principales productores de resinas, aceites, extractos y taninos en Latinoamérica, pero la mayoría de los resultados obtenidos carece de datos en campo y de respuestas acerca del uso práctico y comercial en maderas, y tampoco están catalogados en Brasil. Así, el desarrollo más eficaz de las formulaciones naturales demanda un conjunto de experimentos de laboratorio y también de campo para lograr el éxito de la comercialización. Las dificultades acerca de las nuevas formulaciones deben aún ser superadas y así mejorar a los principales problemas de la comercialización. A pesar del interés que presentan los preservadores naturales, los progresos alcanzados para desarrollar nuevas formulaciones crecen a un ritmo más lento en Brasil que en otros países, donde aún se utilizan productos comerciales que contienen sustancias tóxicas y están vinculados a la contaminación del medio ambiente.

\section{AGRADECIMIENTOS}

Los autores agradecen el apoyo de la Fundação de Amparo à Pesquisa do Estado de São Paulo - FAPESP (Brasil) (Proc. 15/16880-5 y Proc. 18/22413-9), Lemann Institute for Brazilian Studies, University of Illinois at Urbana-Champaign (Estados Unidos de América) y Coordenação de Aperfeiçoamento de Pessoal de Nível Superior - CAPES (Brasil) (Código de financiación 001).

\section{REFERENCIAS}

ABNT (Associação Brasileira de Normas Técnicas, BR). 1997. ABNT NBR 7190. Projeto de estruturas de madeira. $107 \mathrm{p}$. ANVISA (Agência Nacional de Vigilância Sanitária, BR). 2018. C10 - Cipermetrina. Consultado 07 abr. 2020. Disponible en: http://portal.anvisa.gov.br/documents/111215/117782/ C10\%2B\%2BCipermetrina.pdf/37400888-3f11-44edb53f-dea1abacb865

Barbosa AP, CS Nascimento, JW Morais. 2007. Estudos de propriedades antitermíticas de extratos brutos de madeira e casca de espécies florestais da Amazônia Central, Brasil. Acta 
Amazonica 37(2): 213-218. DOI: http://doi.org/c8jvg5

Bertolini MS, FAR Lahr, MF Nascimento, JAM Agnelli. 2013. Accelerated artificial aging of particleboards from residues of CCB treated Pinus sp. and castor oil resin. Materials Research 16(2): 293-303. DOI: http://doi.org/c3n8

Borges CC, GHD Tonoli, TM Cruz, PJ Duarte, TA Junqueira. 2018. Nanoparticles-based wood preservatives: the next generation of wood protection? Cerne 24(4): 397-407. DOI: $\underline{10.1590 / 01047760201824042531}$

Bossardi K, RM Barreiros. 2011. Extrativos naturais como preservantes para madeiras de rápido crescimento - uma revisão. Revista Ciência da Madeira 2: 109-118. DOI: http:// doi.org/c3n9

Brocco VF, JB Paes, LG Costa, S Brazolin, MDC Arantes. 2017. Potential of teak heartwood extracts as a natural wood preservative. Journal of Cleaner Production 142(4): 20932099. DOI: $10.1016 /$ i.jelepro.2016.11.074

Brocco, VF, JB Paes, LG Costa, GT Kirker, S Brazolin. 2020. Wood color changes and termiticidal properties of teak heartwood extract used as a wood preservative. Holzforschung 74(3): 233-245. DOI: 10.1515/hf-2019-0138

Calegari L, JS Porto, DM Nejeliski, LC Duarte, BF Oliveira. 2017. Experimental study on waterproofing MDF with castor oil-based vegetal polyurethane. Matéria 22(3): e11877. DOI: http://doi.org/c3pd

Calegari L, PJG Lopes, GM Santana, DM Stangerlin, E Oliveira, DA Gatto. 2014. Eficiência de extrato tânico combinado ou não com ácido bórico na proteção da madeira de Ceiba pentandra contra cupim xilófago. Floresta 44(1): 43-52. DOI: http://doi.org/c3pc

Cheng SS, J-Y Liu, E-H Chang, S-T Chang. 2008. Antifungal activity of cinnamaldehyde and eugenol congeners against wood-rot fungi. Bioresource Technology 99(11): 51455149. DOI: $\underline{10.1016 / \mathrm{j} . \text { biortech.2007.09.013 }}$

Dias KD, RM Barreiros. 2017a. Potentiality of tall oil as preservative for wood. Chemical and Biomolecular Engineering 2(4): 180-183. DOI: $10.11648 /$ j.cbe.20170204.12

Dias KD, RM Barreiros. 2017b. Resistência à biodeterioração das madeiras de rápido crescimento tratadas com tall oil e derivados. Revista Científica Multidisciplinar Núcleo do Conhecimento 2: 22-36.

Fernandes SR, L Barreiros, RF Oliveira, A Cruz, C Prudêncio, AI Oliveira, C Pinho, N Santos, J Morgado. 2019. Chemistry, bioactivities, extraction and analysis of azadirachtin: State-of-the-art. Fitoterapia. 134: 141-150. DOI: https:// doi.org/10.1016/j.fitote.2019.02.006

Florez JB, PF Trugilho, JT Lima, JRM da Silva. 2014. Caracterización de la madera joven de Tectona grandis L.f. plantada en Brasil. Madera y Bosques 20(1):11-20. DOI: 10.21829/ myb.2014.201172

Goodell B, DD Nicholas, TP Schultz. 2003. Introduction to wood deterioration and preservation. In Goodell B, DD Nicholas, TP Schultz eds. Wood Deterioration and Preservation. Advances in Our Changing World. ACS Symposium Series 845: 2-7. DOI: http://doi.org/bjqqst

Hasan AR, L Hu, HM Solo-Gabriele, L Fieber, Y Cai, TG Townsend. 2010. Field-scale leaching of arsenic, chromium and copper from weathered treated wood. Environmental Pollution 158(5): 1479-1486. DOI: http://doi.org/fbptsn

Hosseinpourpia R, S Adamopoulos, C Parsland. 2019. Utilization of different tall oils for improving the water resistan- ce of cellulosic fibers. Journal of Applied Polymer Science 136(13): 47303(1-8). DOI: 10.1002/app.47303

IBAMA (Instituto Brasileiro do Meio Ambiente e dos Recursos Naturais Renováveis, BR). 2018. Produtos preservativos de madeiras. Consultado 07 abr. 2020. Disponible en: http:// ibama.gov.br/preservativos-de-madeiras/sobre-os-produtos-preservativos-de-madeiras/337-substancias-quimicas-e-biologicas/substancias-quimicas/preservativos-demadeira/989-produtos-preservativos-de-madeiras

Kamdem DP, A Pizzi, A Jermannaud. 2002. Durability of heattreated wood. Holz Als Roh- und Werkstoff 60(1): 1-6. DOI: http://doi.org/dnpfhr

Lepage E, AG Salis, ECR Guedes. 2017. Tecnologia de proteção da madeira. São Paulo, Brasil. Montana Química. 225 p.

Machado GO, C Calil Junior, WL Polito, A Pawlicka. 2006. Preservante natural de madeira para uso na construção civil - óleo de neem. Minerva 3(1): 1-8.

Machado GO, LJ Cookson, AL Christoforo, WL Polito, MR Silva, C Calil Jr, FAR Lahr. 2013. Wood preservation based on neem oil: evaluation of fungicidal and termiticidal effectiveness. Forest Products Journal 63(5-6): 202-206. DOI: http://doi.org/f5nrcm

Medeiros FCM, N. Gouveia, HR Bizzo, RF Vieira, CHS Menezzi. 2016. Fungicidal activity of essential oils from Brazilian Cerrado species against wood decay fungi. International Biodeterioration \& Biodegradation 114: 87-93. DOI: 10.1016/j.ibiod.2016.06.003

Mirski R, D Dziurka, D Dukarska, R Czarnecki, G Cofta. 2014. The influence of microfungi on physicomechanical properties of particle boards. BioResources 9(4): 6329-6339. DOI: http://doi.org/f6sqc3

Nascimento MS, ALBD Santana, CA Maranhão, LS Oliveira, L Bieber. 2013. Phenolic extractives and natural resistance of wood. In Chamy R, F Rosenkranz eds. Biodegradation Life of Science. IntechOpen. p. 349-370. DOI: http://doi. org/c3ph

Oliveira RM, A Brisolari, A Sales, D Gonçalves. 2010. Wettability, shrinkage and color changes of Araucaria angustifolia after heating treatment. Materials Research 13(3): 351-354. DOI: http://doi.org/c8jsqr

Paes JB, AD Souza, CR Lima, PF Souza. 2012. Efficiency of neem (Azadirachta indica A. Juss.) and castor oil plant ( $R i$ cinus communis L.) oils for the improvement of Ceiba pentranda (L.) Gaerth. wood resistance to xilophagous fungi in soil bed test. Ciência Florestal 22(3): 617-624. DOI: http:// doi.org/c3pm

Paes JB, AD Souza, CR Lima, PN Medeiros Neto. 2011. Eficiência dos óleos de nim (Azadirachta indica) e de mamona (Ricinus communis) na proteção da madeira de sumaúma (Ceiba pentandra) contra cupins xilófagos em ensaio de preferência alimentar. Revista Árvore 35(3): 751-758. DOI: http://doi.org/ftwdgh

Papadopoulos AN, HR Taghiyari. 2019. Innovative wood surface treatments based on nanotechnology. Coatings 9(12): 866880. DOI: $10.3390 /$ coatings9120866

Sattolo NMS, D Britto, OBG Assis. 2010. Chitosan as a fungicide for the pinewood used for assembling " $\mathrm{K}$ " type boxes. Brazilian Journal of Food Technology 13(2): 128-132. DOI: http://doi.org/c7kbs5

Shirmohammadl Y, E Davood, AP Efhamisisi. 2018. Tannins as a sustainable raw material for green chemistry: a review. In- 
dustrial Crops and Products 126: 316-332. DOI: $10.1016 / \mathrm{j}$. indcrop.2018.10.034

Silveira AG, EJ Santini, SM Kulczynski, R Trevisan, 2017. Atividade antifúngica do extrato natural da acácia-negra a $P y c$ noporus sanguineus. Scientia Forestalis 45(114): 383-391. DOI: http://doi.org/c3pp

Singh T, AP Singh. 2012. A review on natural products as wood protectant. Wood Science and Technology 46(5): 851-870. DOI: http://doi.org/cnr4v2

Sousa SF, JB Paes, MDC Arantes, YM Lopez, VF Brocco. 2018. Análise física e avaliação do efeito antifúngico dos óleos de andiroba, copaíba e pinhão-manso. Floresta 48(2):153162. DOI: $\underline{10.5380 / \mathrm{rf} . v 48 \mathrm{i} 2.52280}$

Tomak ED, O Gonultas. 2018. The wood preservative potentials of valonia, chestnut, tara and sulphited oak tannins. Journal of Wood Chemistry and Technology 38(3): 183-197. DOI: http://doi.org/c3pr

Viana PA, HT Prates, PEA Ribeiro. 2006. Uso do extrato aquoso de folhas de nim para o controle de Spodoptera frugiperda na cultura do milho. EMBRAPA, Circular Técnica
1-5. Consultado 07 abr. 2020. Disponible en https://www. embrapa.br/documents/1344498/2767891/uso-do-extratoaquoso-de-folhas-de-nim-para-o-controle-de-spodopterafrugiperda-na-cultura-do-milho.pdf/f1d204a5-fa0d-4818b859-59d30d039605

Vidal JM, WV Evangelista, JC Silva, IP Jankowsky. 2015. Preservação de madeiras no Brasil: histórico, cenário atual e tendências. Ciencia Florestal 25(1):257-270. DOI: http:// dx.doi.org/10.5902/1980509817484

Wako SE. 2015. Behaviour and ecological impacts of termites: Fecundity investigations in mounds. Ekologia 34(1): 72-81. DOI: http://doi.org/c3pt

Zablonsky KM, GM Bonduelle, R Trianoski. 2017. Effect of thermal treatment with soybean oil on wood Pinus. Floresta e Ambiente 24: 1-10. DOI: http://doi.org/c3pv

Ziglio AC, MR Sardela, D Gonçalves. 2018. Wettability, surface free energy and cellulose crystallinity for pine wood (Pinus sp.) modified with chili pepper extracts as natural preservatives. Cellulose 25(10): 6151-6160. DOI: $\underline{\text { http:// }}$ doi.org/gfbbrf

Recibido: $05 / 05 / 20$

Aceptado: 17/07/20 